\title{
Fatores condicionantes do desenvolvimento socioeconômico na América Latina: uma análise sob a perspectiva do PIB e dos indicadores globais de governança
}

Conditioning factors of socioeconomic development in Latin America: an analysis from the perspective of PIB and the global governance indicators

\section{Facteurs influant sur le développement socio-économique en Amérique latine: une analyse dans la perspective du PIB et des indicateurs de gouvernance mondiale}

\author{
Factores condicionantes del desarrollo socioeconómico en América latina: un
} análisis desde la perspectiva del PIB y de los indicadores globales de gobernanza

\author{
Francisco Aquiles de Oliveira Caetano ${ }^{1}$ \\ Jair Andrade de Araújo \\ Ahmad Saeed Khan
}

Recebido em 03/07/2017; revisado e aprovado em 20/12/2017; aceito em 27/12/2017

DOI: http://dx.doi.org/10.20435/inter.v0i0.1646

\begin{abstract}
Resumo: O objetivo deste artigo foi ampliar a discussão sobre a relação entre o desenvolvimento socioeconômico e os indicadores de governança na América Latina, entre 2000 e 2014. Quanto aos aspectos metodológicos, empregou-se o modelo de regressão com dados em painel utilizando dados secundários. O uso da modelagem de dados em painel permitiu identificar impactos positivos das boas práticas de governança e do Produto Interno Bruto (PIB) sobre o desenvolvimento socioeconômico, e impactos negativos da Qualidade Regulatória em todos os cenários estimados.
\end{abstract}

Palavras-chave: desenvolvimento socioeconômico; indicadores de governança; América Latina.

Abstract: The aim of this study was analyse the relationship between socioeconomic development and governance indicators in Latin America between 2000 and 2014. It was conducted a regression model with panel data using secondary data. The panel data modeling allowed the identification of positive impacts of good governance and gross domestic product (GDP) practices on socioeconomic development and negative impacts of Regulatory Quality in all the estimated scenarios.

Keywords: socioeconomic development; governance indicators; Latin America.

Résumé: L'objectif de cet article était d'élargir la discussion sur la relation entre le développement socioéconomique et les indicateurs de gouvernance en Amérique Latine, entre 2000 et 2014 . En ce qui concerne les aspects méthodologiques, le modèle de régression a été utilisé avec des données de panel avec des données secondaires. L'utilisation de la modélisation des données de panel a permis d'identifier les impacts positifs de les bonnes pratique de gouvernance et du PIB sur le développement socio-économique et les impacts négatifs de la Qualité Réglementaire dans tous les scénarios estimés.

Mots-clés: developpement socio-economique; indicateurs de gouvernance; Amérique Latine.

Resumen: El objetivo de este artículo fue ampliar la discusión sobre la relación entre el desarrollo socioeconómico y los indicadores de gobernanza en América Latina entre 2000 y 2014 . En cuanto a los aspectos metodológicos, se utilizó el modelo de regresión con datos en panel con datos secundarios. La utilización del modelado de datos en panel permitió identificar impactos positivos de las buenas prácticas de gobernanza y del PIB sobre el desarrollo socioeconómico y los impactos negativos de la Calidad Regulatoria en todos los escenarios estimados.

Palabras clave: desarrollo socioeconómico; indicadores de gobernanza; América Latina.

\footnotetext{
${ }^{1}$ Universidade Federal do Ceará (UFC), Campus do Pici, Fortaleza, Ceará, Brasil.
} 


\section{INTRODUÇÃO}

A globalização, as novas tecnologias e o maior acesso a informações têm possibilitado à sociedade um maior contato com as rápidas transformações do meio em que se vive. Com isso, tornou-se possível um maior incremento no capital social das nações e, consequentemente, uma maior participação de stakeholders ${ }^{2}$ na vida pública de seus países. Nesse contexto, em que a sociedade busca compreender e participar das transformações políticas, os princípios de governança surgem como fator condicionante do desenvolvimento humano e do bem-estar social.

Há evidências empíricas de que existe uma relação entre a boa governança e um melhor resultado no desenvolvimento, como maiores rendimentos per capita, menor mortalidade infantil e maior alfabetização (KAUFMANN; KRAAY; ZOIDO-LOBATÓN, 1999). Grindle (2004) afirma que países desenvolvidos apresentam princípios de governança mais consistentes porque foram embasados em outros que os precederam, evidenciando que a utilização de boas práticas de governança deve ser assumir um caráter de processo contínuo para gerar resultados eficazes.

Contudo há uma complexidade na relação entre a governança e o desenvolvimento. Isso se explica, em grande parte, pela imprecisão na mensuração de seus conceitos, ambos multidimensionais (STURM, 2013). O entendimento de governança sofreu diversas alterações, adaptações e aplicações no decorrer do tempo. Inicialmente, na década de 1980, associado a processos administrativos gerenciais, a governança passou a considerar a interação entre a sociedade civil, as instituições públicas e o terceiro setor. De maneira geral, a governança é definida como as tradições e instituições pelas quais a autoridade de um país é exercida, observando o processo pelo qual os governos são selecionados, monitorados e substituídos, a capacidade do governo de formular e implementar políticas sólidas e o respeito dos cidadãos e do Estado às instituições que governam suas interações econômicas e sociais (WORLD BANK, 2017a).

Já o desenvolvimento, quase sempre confundido com crescimento econômico, pode ser medido pela vertente do crescimento da renda (Produto Interno Bruto [PIB] ou PIB per capita) ou pela vertente dos ganhos em qualidade de vida e bem-estar, como aborda o Índice de Desenvolvimento Humano (IDH). O IDH inclui em sua análise a expectativa de vida, quantidade média de anos de estudo e anos esperados de escolaridade, e o Produto Nacional Bruto (PNB), como proxies de saúde, educação e renda, respectivamente.

Há claras evidências de que o desempenho econômico dos países possui grande influência sobre o seu desenvolvimento humano, contudo os determinantes desse desenvolvimento são muito heterogêneos e o Produto Interno Bruto (PIB) pode não ser o principal fator dessa condição (GAYGISIZ, 2013). Diante da necessidade de identificar os demais fatores que influenciam o desenvolvimento socioeconômico, a presente proposta se embasa nos estudos de Rajkumar e Swaroop (2008) , Sturm (2013) e Gaygisiz (2013) para verificar se os Indicadores de Governança Global (Worldwide Governance Indicators [WGI], em inglês) possuem poder de explicação para esse progresso.

Nessa perspectiva, diante da necessidade em avançar no estudo da governança, a partir da década de 1990, foi desenvolvido um grande número de indicadores para aferi-la, contudo

\footnotetext{
${ }^{2}$ Qualquer pessoa, grupo ou entidade que tenha interesse nas atividades, recursos ou resultados de uma entidade do sector público, ou que seja afetada por essa produção. Stakeholders podem ser também reguladores, acionistas, funcionários, clientes, fornecedores, grupos de defesa, governos, parceiros de negócios e a sociedade como um todo (INTERNATIONAL FEDERATION OF ACCOUNTANTS [IFAC], 2013).
} 
os WGI, do Banco Mundial, tornaram-se os mais consagrados nos estudos de governança em nível mundial (JACQUES; VICENTE; ENSSLIN, 2013).

O presente estudo tem como objetivo analisar a relação entre o desenvolvimento humano, a renda e os indicadores de governança global (WGI). Diante disso, este artigo se propõe a investigar a seguinte questão: qual o impacto do crescimento econômico e dos indicadores de governança global (WGI) sobre o desenvolvimento socioeconômico (IDH)? Ademais, parte-se da hipótese de que os indicadores de governança mundial (WGI) afetam positivamente o desempenho do desenvolvimento humano dos países da América Latina.

O escopo do estudo é fundamentado na relevância da investigação dos impactos dos indicadores de governança no desenvolvimento de países latino-americanos, que passaram por um período de grande crescimento a partir da década de 2000, contudo tem se caracterizado pela distribuição desigual de recursos, não refletindo em grandes avanços no desenvolvimento e no bem-estar social.

\section{GOVERNANÇA GLOBAL E SOCIEDADE}

A etimologia da palavra governança evidencia uma origem no grego antigo e no latim clássico associado à "direção", inicialmente ligado à ação ou ao modo de governar, guiar ou conduzir (JESSOP, 1998). O termo governança passou a receber uma nova interpretação nos anos 1980 e 1990, quando passou a referir-se à modernização de processos administrativos empresariais, passando a ser tratada por Governança Corporativa. Inicialmente utilizada para fazer referência a processos corporativos de administração, passou a designar uma abertura para participação de setores e atores da sociedade civil nos processos políticos (MELO NETO e BRENNAND, 2004).

Os governos, por meio de suas instâncias representativas, começaram a ser exigidos no sentido de promover a integração da participação popular e do setor privado na gestão pública (VIANA, 2013). O Estado, que já demonstrava uma propensão à concepção neoliberal de Estado mínimo, além de defender a regulação pelo mercado, mostrou-se inclinado a uma maior cooperação popular e à efervescência da busca pelo capital social (WEISS, 2000).

$O$ atual entendimento da governança como conceito nas ciências sociais abrange contextos mais amplos do que a sua interpretação como sinônimo de "governo", apresentando múltiplos significados e implicações (KJAER, 2004). Essa concepção moderna de governança utiliza uma abordagem sistêmica, em que todo o processo tem importância, da concepção aos resultados, e permite envolver todos os interessados, considerando as demandas individuais e de stakeholders. Em seu relatório, a Comissão sobre Governança Global (1996) afirma que a governança engloba todas as diversas maneiras pelas quais os indivíduos e as instituições, públicas e privadas, administram seus problemas comuns, por meio de um processo contínuo que acomode interesses conflitantes ou diferentes, de forma a realizar ações cooperativas.

A literatura acadêmica, principalmente os cientistas sociais, tem mostrado um interesse no estudo da governança, em decorrência da preocupação com a sua influência sobre a mudança na maneira de governar (STOKER, 1998). Diante disso, o Quadro 1 apresenta as principais definições de governança. 
Quadro 1 - Principais definições de governança

\begin{tabular}{|c|c|}
\hline Definição & Autor \\
\hline $\begin{array}{l}\text { Governança em geral é a definição, aplicação e execução das regras do jogo. } \\
\text { Governança refere-se à auto-organização, a redes interorganizadas } \\
\text { caracterizadas pela independência, troca de recursos, regras do jogo e } \\
\text { autonomia significativa do Estado. }\end{array}$ & (RHODES, 1997) \\
\hline $\begin{array}{l}\text { Governança é a capacidade institucional das organizações públicas de } \\
\text { fornecer o público e outros bens exigidos pelos cidadãos de um país ou } \\
\text { seus representantes de forma eficaz, transparente, imparcial e responsável, } \\
\text { sujeito a restrições de recursos. }\end{array}$ & (WORLD BANK, 2000) \\
\hline $\begin{array}{l}\text { Governança refere-se à criação de uma estrutura ou uma ordem, que } \\
\text { não pode ser imposta externamente, e resulta da interação de uma } \\
\text { multiplicidade de atores influenciando uns aos outros na condução dos } \\
\text { problemas coletivos. }\end{array}$ & $\begin{array}{l}\text { (KOOIMAN; VAN } \\
\text { VLIET, 1993) }\end{array}$ \\
\hline $\begin{array}{l}\text { Governança compreende os mecanismos (político, econômico, } \\
\text { socioambiental, administrativo, legal, entre outros) colocados em prática } \\
\text { para garantir que os resultados pretendidos para as partes interessadas } \\
\text { sejam definidos e alcançados. }\end{array}$ & (IFAC, 2013) \\
\hline
\end{tabular}

Fonte: Elaboração própria

Os princípios da governança reforçam que esse processo tem como fim produzir resultados eficazes, não estando apenas restrito ao seu planejamento. Para Finkelstein (1991), o estudo da governança não se restringe apenas a decisões, mas também às suas consequências, como efeitos distributivos, eficácia, programas e projetos, consentimento e implementação doméstica. Timmers (2000) afirma que o resultado esperado na Governança Pública é a melhoria dos serviços prestados à sociedade e dos benefícios obtidos pela população.

A governança tem sido referência na elaboração e efetivação de políticas de interesse global, como políticas ambientais e de desenvolvimento. A participação de organismos internacionais como a ONU, o Fundo Monetário Internacional (FMI) e o Banco Mundial, intermediando temas de interesse de mundiais, têm chamado a atenção para uma Governança Global. Desde o ano de 1996, o Banco Mundial coleta e publica dados de governança de mais de 200 países, por meio do Worldwide Governance Indicators (WGI), que mede seis dimensões de governança, representados pelos indicadores Voz e Responsabilidade (VR), Estabilidade Política e Ausência de Violência (EPAV), Eficácia Governamental (EG), Qualidade Regulatória (QR), Estado de Direito (ED) e Controle da Corrupção (CC).

Na visão de Kaufmann e Kraay (2008), os indicadores de governança têm grande importância, pois funcionam como agregadores de diversos outros indicadores individuais que se complementam, aumentando a precisão dos resultados obtidos. Wolfensohn (1998) afirma que a importância da boa governança para o desenvolvimento alcançou o status de critério formal, vista a importante relação entre estes fatores. Logo, as seis dimensões do WGI podem funcionar como proxies de governança, auxiliando possíveis reformas do setor público.

A instabilidade política e os baixos índices de desenvolvimento dos países da América Latina, foco do presente estudo, justificam uma análise do comportamento dos indicares de governança dessas localidades, que historicamente possuem níveis de crescimento econômico e de desenvolvimento humano menores que o nível mundial (RANIS; STEWART, 2002).

Nas últimas décadas, evidenciou-se que o desenvolvimento humano apresenta relação com a estabilidade político-governamental de uma nação (UNITED NATIONS DEVELOPMENT 
PROGRAMME [UNDP], 1997; KAUFMANN; KRAAY, 2008; KAUFMANN; KRAAY; ZOIDOLOBATÓN, 1999). Segundo Moura (2014), a prática da boa governança é o elo necessário entre o desenvolvimento e as ações governamentais, visto que seus princípios deveriam aumentar a eficiência e a legitimidade nos processos de elaboração e execução de políticas públicas.

A América Latina passou por um período de grande crescimento a partir da década de 2000, contudo tem se caracterizado distintivamente pela distribuição desigual de recursos, um obstáculo para o bem-estar atual e futuro desenvolvimento de suas sociedades e economias (COMISSÃO ECONÔMICA PARA A AMÉRICA LATINA E CARIBE [CEPAL], 2015). Se considerada a particular relação entre a governança e o desenvolvimento humano, depreende-se que o crescimento econômico observado nos últimos anos não foi acompanhado da aplicação dos princípios que caracterizam a boa governança.

\section{CRESCIMENTO ECONÔMICO E DESENVOLVIMENTO SOCIOECONÔMICO}

O crescimento econômico, ou o crescimento da renda per capita, é comumente tratado como sinônimo do desenvolvimento social e econômico das nações, contudo o crescimento da renda é apenas uma vertente do incremento do padrão de vida da população. Schumpeter (1961) foi um dos primeiros a observar que o crescimento da renda per capita não assegurava o desenvolvimento, afirmando ser necessário que haja mudanças estruturais no sistema econômico. A partir de 1990, a ONU (Organização das Nações Unidas) iniciou a publicação do Human Development Report (HDR), elaborado pelos economistas Mahbub ul Haq e Amartya Sen, focando em uma nova abordagem de desenvolvimento que inclui as dimensões social, cultural, política, econômica e ambiental.

O desenvolvimento humano é um processo de ampliação das escolhas e oportunidades das pessoas, incluindo viver uma vida longa e saudável, ser educado e ter acesso a recursos que são essenciais para alcançar um padrão de vida decente (UNDP, 1990). O aumento do desenvolvimento humano das nações implica satisfação das necessidades e aumento da produtividade e eficiência da população impactada, criando um ambiente propício para o crescimento econômico. Amartya Sem (1997) afirma que o papel do crescimento econômico é integrado à compreensão fundamental do processo de desenvolvimento humano como a expressão de uma maior liberdade e melhor qualidade de vida.

Ao analisar a América Latina, Ranis e Stewart (2002) destacaram que há uma série de relações causais entre o crescimento econômico e o desenvolvimento humano nesses países, contudo essas conexões não são automáticas, visto que dependem, dentre outros fatores, das opções políticas. Yang (2010) afirma que a forma como as políticas são feitas e aplicadas é determinada pela qualidade e desempenho das administrações políticas.

A qualidade das instituições é um importante fator para o desenvolvimento humano, visto que as sociedades são mais propensas a operar melhor e mais eficientemente sob governos eficazes (YANG, 2010). O autor destaca que os países desenvolvidos apresentam em comum, em diferentes níveis, instituições governamentais eficazes e eficientes.

Diante dessa observação, a governança emergiu como um indicador de medida da qualidade institucional (KAUFMAN; KRAAY, 2008), e suas dimensões possuem relação direta com o desempenho da economia. Infere-se que a robustez de indicadores de governança, como Estabilidade Política e Estado de Direito, tende a impactar positivamente o crescimento econômico. 


\section{MATERIAL E MÉTODOS}

Os indicadores globais de governança (WGI) são captados desde o ano de 1996, abrangendo mais de 200 países e territórios. Os seis indicadores agregados de governança são a sintetização de centenas de variáveis individuais subjacentes, captadas de diversas fontes, e são apresentados anualmente pelo Banco Mundial. De forma complementar, junto aos indicadores de governança, foram captados os dados do Produto Interno Bruto (PIB), total e per capita (WORLD BANK, 2017).

Os dados referentes ao Índice de Desenvolvimento Humano (IDH) foram aferidos junto aos Relatórios de Desenvolvimento Humano (HDR), publicados anualmente (UNDP, 2015) e utilizados como proxies do desenvolvimento socioeconômico.

As variáveis utilizadas no estudo, sua representação e sua descrição estão sumarizadas no Quadro 2.

Quadro 2 - Variáveis utilizadas no estudo da Governança e do Desenvolvimento nos países da América Latina

\begin{tabular}{|c|c|c|}
\hline Representação & Variável & Descrição \\
\hline IDH & $\begin{array}{l}\text { Índice de Desenvolvimento } \\
\text { Humano }\end{array}$ & $\begin{array}{l}\text { Mede o desenvolvimento de uma região baseado em } \\
\text { três fatores: educação, expectativa de vida e renda. }\end{array}$ \\
\hline $\mathrm{PIBpc}$ & PIB per capita (US\$) & $\begin{array}{l}\text { Mede o Produto Interno Bruto dividido pela } \\
\text { população de um país. }\end{array}$ \\
\hline PIBt & PIB total (US\$) & $\begin{array}{l}\text { Mede, em valores monetários, a soma de todos os } \\
\text { bens e serviços finais produzidos por um país. }\end{array}$ \\
\hline WGIvr & Voz e Responsabilização & $\begin{array}{l}\text { Mede a percepção de quanto o cidadão de um país é } \\
\text { capaz de participar na escolha de seu governo, bem } \\
\text { como sua liberdade de expressão e associação, e a } \\
\text { existência de uma imprensa livre. }\end{array}$ \\
\hline WGlep & $\begin{array}{l}\text { Estabilidade Política e } \\
\text { Ausência de Violência }\end{array}$ & $\begin{array}{l}\text { Mede a percepção do risco de um governo } \\
\text { ser desestabilizado ou deposto por meios } \\
\text { inconstitucionais ou violentos, incluindo violência } \\
\text { politicamente motivada ou terrorismo. }\end{array}$ \\
\hline WGleg & Eficácia do Governo & $\begin{array}{l}\text { Mede a percepção da qualidade do serviço público } \\
\text { e seu grau de independência de pressões políticas, a } \\
\text { qualidade da elaboração e implementação, bem comc } \\
\text { o comprometimento do governo com essas políticas. }\end{array}$ \\
\hline WGIqr & Qualidade Regulatória & $\begin{array}{l}\text { Mede a percepção da habilidade de um governo } \\
\text { para formular e implementar políticas e leis sólidas, } \\
\text { capazes de promover o desenvolvimento do setor } \\
\text { privado. }\end{array}$ \\
\hline WGled & Estado de Direito & $\begin{array}{l}\text { Mede a percepção da confiança que os agentes têm e } \\
\text { o quanto eles respeitam as regras sociais, em especial } \\
\text { a qualidade da execução dos contratos, os direitos de } \\
\text { propriedade, a polícia e os tribunais. }\end{array}$ \\
\hline WGIcc & Controle da Corrupção & $\begin{array}{l}\text { Mede a percepção de quanto o poder público é } \\
\text { exercido para ganhos particulares, quer sejam } \\
\text { pequenas ou grandes manifestações de corrupção, } \\
\text { assim como a parcela do Estado que é capturada pela } \\
\text { elite e interesses privados. }\end{array}$ \\
\hline
\end{tabular}

Fonte: World Bank (2017a). 


\subsection{Procedimentos metodológicos}

O método utilizado para a análise dos dados é o modelo de regressão com dados em painel com informações agregadas para países da América Latina. A análise considera o desenvolvimento socioeconômico e o crescimento econômico no período de 2004 a 2014, e como determinante as variáveis de governança, representadas pelas dimensões do WGI: Voz e Responsabilização, Estabilidade Política, Eficácia do Governo, Qualidade Regulatória, Estado de Direito e Controle da Corrupção.

Nesse sentido, a estratégia econométrica adotada (modelo com dados em painel), apresenta algumas vantagens interessantes, como a maior quantidade de informação, maior variabilidade dos dados, menor colinearidade entre as variáveis, maior número de graus de liberdade e maior eficiência (BALTAGI, 2005)

\subsubsection{Dados em Painel}

A estimação que relaciona desenvolvimento humano e princípios de boa governança foi efetuada pelo modelo de dados em painel, devido à presença de uma dimensão espacial e uma dimensão temporal nos dados da pesquisa, ou seja, uma mesma unidade de cross-section (corte transversal) será analisada ao longo de um período de tempo estabelecido.

Segundo Klevmarken (1989), Hsiao (2003) e Baltagi (2005), o método de dados em painel apresenta como vantagem a capacidade de identificar e medir melhor os efeitos que não podem ser observados em análises de cross-section puro ou em uma série temporal pura. Arellano (2003) destaca ainda a possibilidade de seguir os mesmos indivíduos ao longo do tempo, facilitando a análise das respostas dinâmicas e o controle da heterogeneidade não-observada.

Segundo Wooldridge (2010), o modelo de dados em painel deve ser representado da seguinte forma:

$$
\mathrm{Y}_{\mathrm{it}}=\mathrm{X}_{\mathrm{it}}^{\prime} \beta+\mathrm{c}_{\mathrm{i}}+\mathrm{u}_{\mathrm{it}} \quad \mathrm{i}=1, \ldots, \mathrm{N} \quad \mathrm{t}=1, \ldots, \mathrm{T}
$$

onde $\mathrm{c}_{\mathrm{i}}$ é uma variável latente que corresponde à heterogeneidade não observada no modelo, tentando reproduzir as características não observáveis entre as observações no crosssection. Ademais, a análise de Wooldridge supõe que o efeito parcial de $\mathrm{c}_{\mathrm{i}}$ é constante no tempo, mas pode variar entre as observações no cross-section.

No presente estudo, a variável latente $c_{i}$ retrata características não observáveis dos diferentes países em análise, englobando fatores de natureza econômica, social, cultural e ambiental, que se somam na determinação da variável dependente, entretanto não são considerados no conjunto de variáveis explicativas, por não terem notória observância ou não serem mensuráveis. Desse modo, a equação (1) pode ser reescrita da seguinte maneira:

$$
\mathrm{Y}_{\mathrm{it}}=\alpha_{\mathrm{i}}+\beta \mathrm{X}_{\mathrm{it}}+\mathrm{c}_{\mathrm{i}}+\mathrm{u}_{\mathrm{it}}
$$

Tem-se que $\boldsymbol{c}_{\mathrm{i}}$ deve variar apenas entre países, e não ao longo do tempo. Ou seja, $\boldsymbol{c}_{\mathrm{i}}$ exprime toda a heterogeneidade não observada associada ao país i, apresentando-se constante ao longo de todo o período amostral. 
A decisão pelo método de estimação mais adequado, se o modelo de efeitos fixos (EF) ou de efeitos aleatórios (EA), depende em grande parte da pressuposição sobre a não correlação entre o componente de erro $\mathrm{u}_{\mathrm{it}}$ e os regressores de $x_{i}$, em qualquer instante de tempo, ou seja, a não-correlação entre $\mathrm{u}_{\mathrm{it}} \mathrm{e} \mathrm{x}_{\mathrm{i} 1}, \ldots, \mathrm{x}_{\mathrm{i}}$. No caso da pressuposição de que há correlação, $\mathrm{o}$ modelo mais adequado pode ser o de EF; contudo, se a suposição for de não correlação, o mais indicado é o de EA.

\section{RESULTADOS E DISCUSSÃO}

As estatísticas rotineiras foram aplicadas com o objetivo de decidir entre o modelo de estimação mais adequado. Utilizou-se, inicialmente, o teste de Chow, que permite escolher entre modelo Pooled e o modelo de Efeitos fixos. O resultado desse teste permitiu rejeitar a hipótese nula, a um nível de $1 \%$ de significância, ou seja, o modelo de efeitos fixos é mais adequado que o modelo Pooled. Diante da existência de efeitos específicos, foi realizado o teste de Hausman, que, por sua vez, permitiu aceitar a hipótese nula de que a diferença entre os estimadores de efeitos aleatórios e os de efeitos fixos seja considerável. Esse resultado indica que o melhor modelo a ser utilizado é o de Efeitos Aleatórios, que será analisado adiante.

O estudo analisou dados do IDH, a taxa de crescimento do PIB e das seis dimensões que compõem os WGI para os países da América Latina, no período que compreende os anos de 2002 a 2014.

O IDH varia de 0 a 1 e, quanto mais próximo de 1 , mais desenvolvida é a nação. Os países e territórios são ranqueados e categorizados como possuidores de Desenvolvimento Humano Muito Alto (0,800 a 1), Alto Desenvolvimento Humano (0,700 a 0,799), Médio Desenvolvimento Humano $(0,555$ a 0,699) ou Baixo Desenvolvimento Humano (menor que 0,555). A composição desse índice inclui a expectativa de vida, quantidade média de anos de estudo e anos esperados de escolaridade, e o Produto Nacional Bruto (PNB), como proxies de saúde, educação e renda, respectivamente.

A evolução do IDH nos países da América Latina, tendo como referência os anos de 2000 e 2014, pode ser observada na Figura 1. No ano 2000, seis países apresentaram alto desenvolvimento humano (Argentina, Chile, Cuba, Costa Rica, Panamá e Uruguai), treze foram classificados como países de médio desenvolvimento humano (Bolívia, Brasil, Colômbia, El Salvador, Equador, Guatemala, Honduras, México, Nicarágua, Paraguai, Peru, República Dominicana e Venezuela) e um com baixo desenvolvimento humano (Haiti). Em 2014, dois países apresentaram desenvolvimento humano muito alto (Argentina e Chile), onze países mostraram-se com alto desenvolvimento humano (Uruguai, Panamá, Cuba, Costa Rica, Venezuela, México, Brasil, Peru, Equador, Colômbia e República Dominicana), seis países indicaram médio desenvolvimento humano (Paraguai, El Salvador, Bolívia, Nicarágua, Guatemala e Honduras) e um país apresentou baixo desenvolvimento humano (Haiti) (UNDP, 2015). 
Figura 1 - Mapa do IDH da América Latina de 2000 e 2014
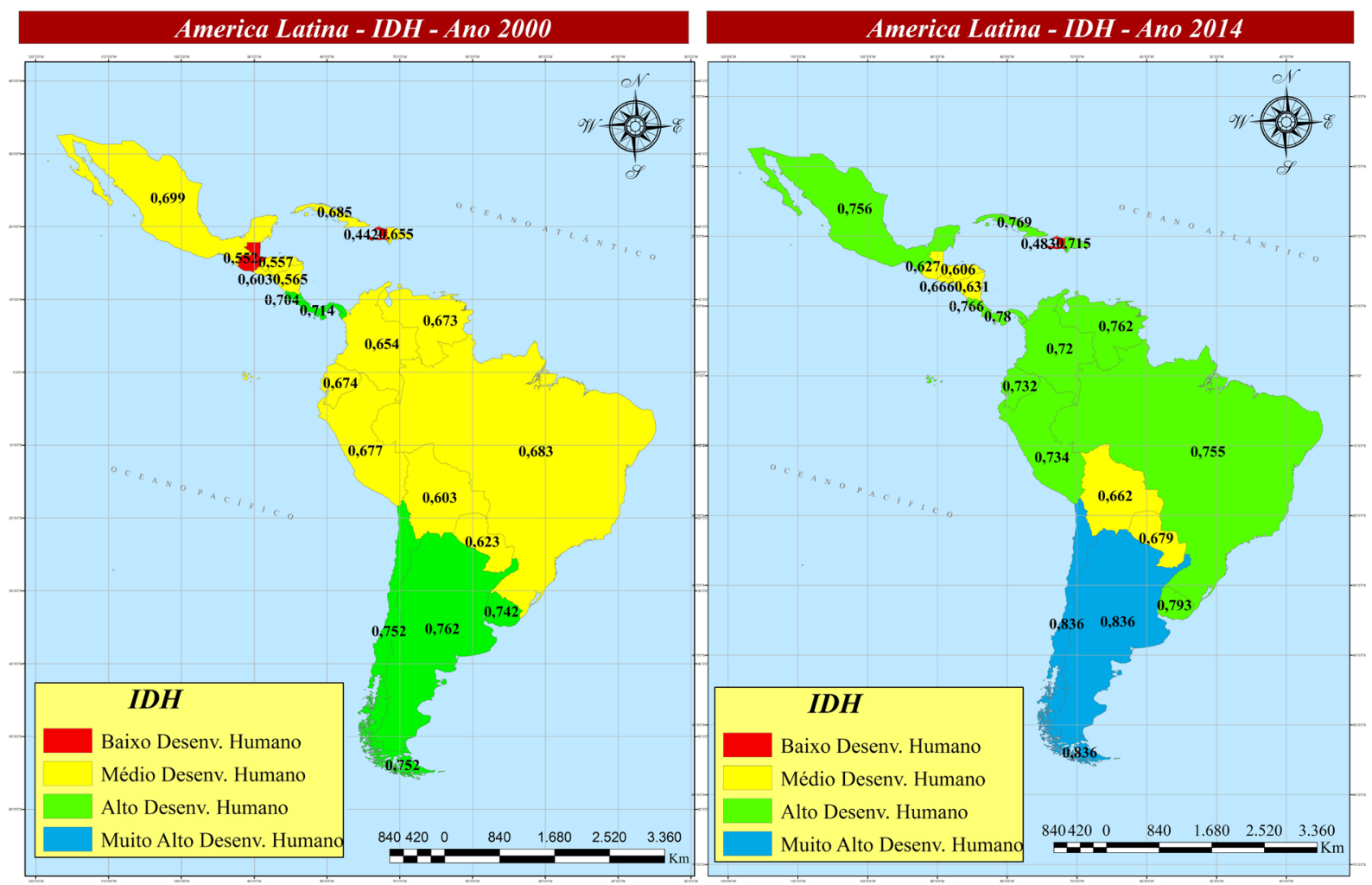

Fonte: Elaboração própria segundo dados da pesquisa.

O IDH apresentou um crescimento uniforme no período que compreende os anos de 2000 a 2014. Essa variável oscilou positivamente entre 6,9\% e 13,6\%, com destaque positivo para Guatemala e Venezuela, que apresentaram crescimento superior a 13\% no período de 15 anos que compreende a pesquisa. Os menores crescimentos acumulados do IDH foram de Uruguai, México e Peru (6,9\%, 8,2\% e 8,4\%, respectivamente)

A Figura 2 analisa o crescimento médio anual do PIB entre 2000 e 2014. É possível observar que Panamá e Peru apresentaram poucas oscilações e obtiveram os melhores resultados médios do período analisado (6,2\% e 5,3\%, respectivamente). Países como República Dominicana (4,9\%), Cuba $(4,6 \%)$, Colômbia (4,3\%), Equador (4,3\%), Costa Rica $(4,1 \%)$, Honduras $(4,1 \%)$, Chile $(4 \%)$ e Paraguai $(3,8 \%)$ também conseguiram resultados acima da média de crescimento da América Latina, que se manteve em $3,7 \%$. As menores taxas de crescimento formam apresentadas por Haiti (1,2\%), El Salvador (1,9\%), México (2,3\%) e Argentina (2,7\%) (WORLD BANK, 2017b). 
Figura 2 - Crescimento médio anual do PIB entre 2000 e 2014

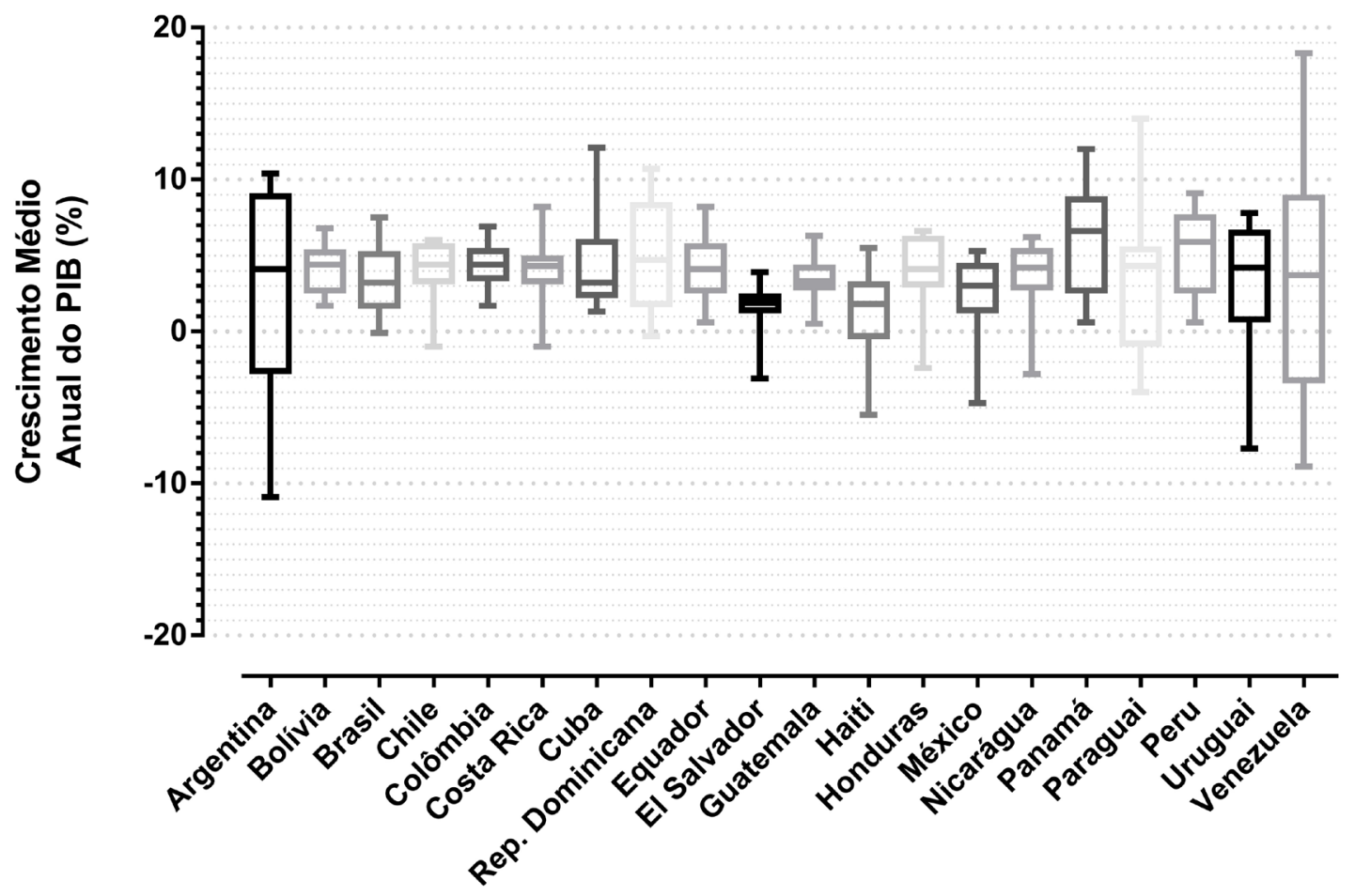

Fonte: Elaboração própria segundo dados da pesquisa.

No ano de 2009, em decorrência do impacto causado pela crise internacional de 2008, houve retração do PIB na maioria das economias da região, com exceção do Haiti. Esse impacto se refletiu também no PIB per capita de 2009, interrompendo o crescimento positivo dessa variável de anos anteriores. O desempenho do PIB per capita dos países da América Latina pode ser observado na Figura 3.

A renda é um dos fatores determinantes do desenvolvimento, e a análise do PIB per capita dos países da latino-americanos demonstra isso. Em 2014, os quatro países com maior PIB per capita da região (Uruguai, Chile, Panamá e Argentina) eram os quatro países classificados como os mais elevados no índice de desenvolvimento humano.

A análise agregada do PIB per capita, de 2000 a 2014, indica com destaque o crescimento de Equador (343\%), Peru (225\%), Colômbia (220\%), Brasil (214\%), Panamá (212\%) e Bolívia (210\%). Os menores crescimentos acumulados foram de México e Argentina (55\% e 60\%, respectivamente). Foi observado que houve uma tendência de menores crescimentos da renda per capita para o grupo de países que possuía melhor desempenho dessa variável no ano 2000. 
Figura 3 - Valores anuais do PIB per capita de países da América Latina entre 2000 e 2014

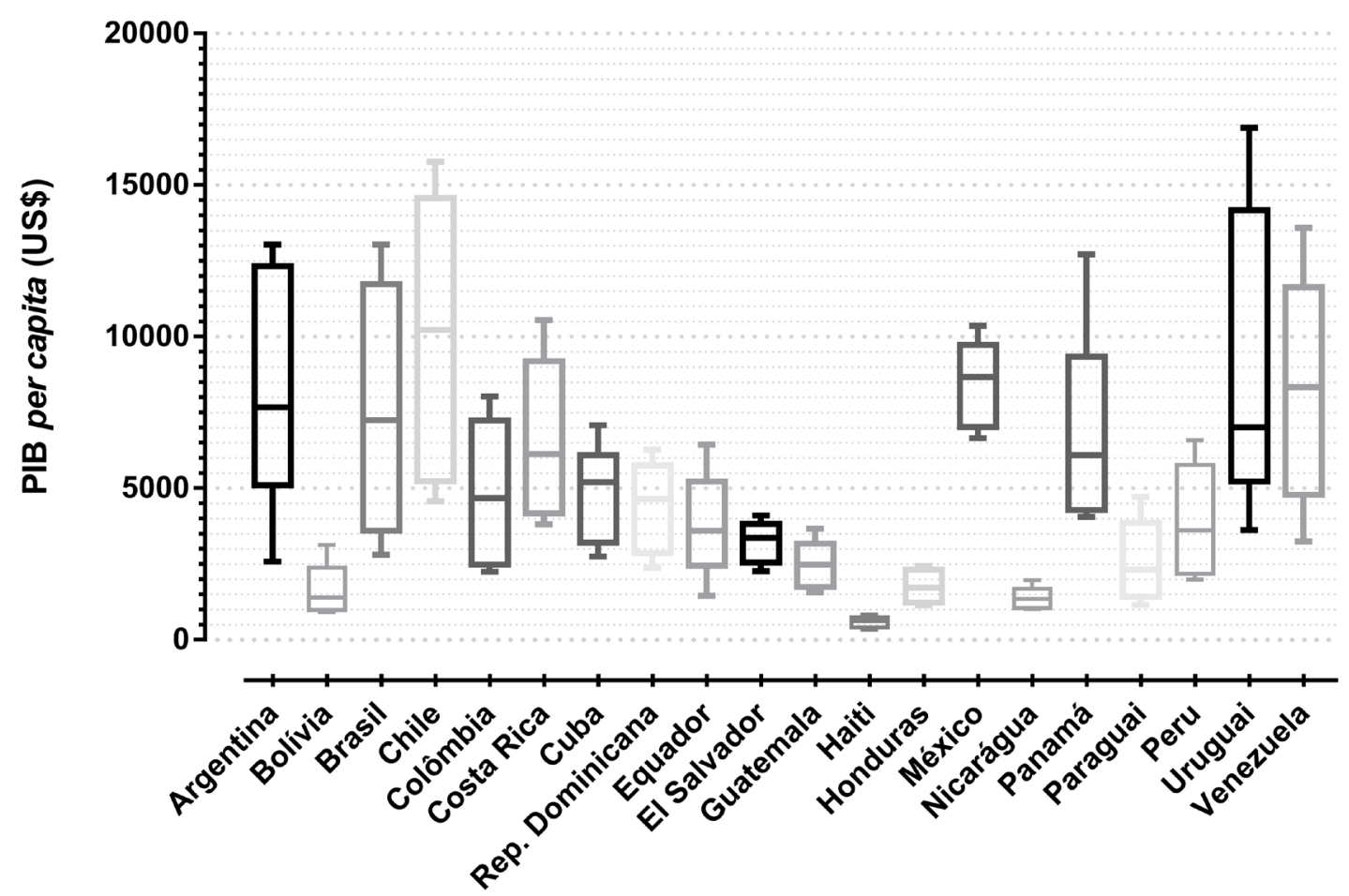

Fonte: Elaboração própria segundo dados da pesquisa

Foram estimadas três especificações com o modelo de dados em painel, via efeitos aleatórios, tendo como variável dependente o Índice de Desenvolvimento Humano dos países latino-americanos. As variáveis explicativas utilizadas na primeira especificação foram as seis dimensões de governança global aferidas pelo Banco Mundial. Nas segunda e terceira especificações, foram adicionadas separadamente as variáveis PIB per capita e PIB total, com o objetivo de testar a sensibilidade dos resultados e dos coeficientes estimados dessas variáveis inclusas no modelo. Os resultados estão expostos na Tabela 1.

Tabela 1 - Resultado da estimação via Efeitos Aleatórios

\begin{tabular}{|c|c|c|c|c|c|c|}
\hline \multirow{2}{*}{ Variável Dependente } & \multicolumn{2}{|c|}{ (1) IDH } & \multicolumn{2}{|c|}{ (2) IDH } & \multicolumn{2}{|c|}{ (3) IDH } \\
\hline & Coef. & P-valor & Coef. & P-valor & Coef. & P-valor \\
\hline Eficácia do Governo & 0.0440 & 0.000 & 0.0148 & 0.040 & 0.0400 & 0.000 \\
\hline $\begin{array}{l}\text { Estabilidade Política e Ausência de } \\
\text { Violência }\end{array}$ & 0.0187 & 0.002 & 0.0076 & 0.052 & 0.0222 & 0.000 \\
\hline Qualidade Regulatória & -0.0475 & 0.000 & -0.0216 & 0.000 & -0.0333 & 0.000 \\
\hline Estado de Direito & 0.0267 & 0.014 & 0.0119 & 0.081 & 0.0054 & 0.586 \\
\hline Voz e Responsabilização & -0.0004 & 0.973 & -0.0026 & 0.724 & -0.0057 & 0.584 \\
\hline Controle da Corrupção & 0.0014 & 0.875 & 0.0003 & 0.958 & 0.0119 & 0.146 \\
\hline PIB per capita & & & 7.37e-06 & 0.000 & & \\
\hline PIB total & & & & & $5.20 e-14$ & 0.000 \\
\hline Constante & 0.7124 & 0.000 & 0.6585 & 0.000 & 0.6964 & 0.000 \\
\hline
\end{tabular}

Fonte: Elaboração própria segundo dados da pesquisa. 
Na primeira especificação, que estima os valores considerando apenas as dimensões de governança como variáveis explicativas, as variáveis Estabilidade Política e Auusência de Violência, Eficácia do Governo e Qualidade Regulatória, apresentaram-se significativas a 1\%. Os resultados indicam que quanto maior for a eficácia do governo e a estabilidade política do país, maior será o seu desenvolvimento humano. Tem-se também uma relação positiva entre o Estado de Direito e o desenvolvimento humano, a uma significância de 5\%. A constante, que representa os efeitos não captados pelo modelo, apresentou variação entre 0,66 a 0,71.

A análise das demais especificações apresenta resultados com valores próximos para a eficácia do governo. Na equação (1) e na equação (3), o coeficiente que representa a eficácia do governo é 0,04 , contudo, na especificação (2), esse coeficiente se apresenta em 0,01. Logo, caso haja um crescimento de $1 \%$ na eficácia do governo, haverá um aumento no desenvolvimento humano do país de aproximadamente 0,04\%. Esse resultado corrobora com a análise de Kaufmann, Kraay e Zoido-Lobatón (1999), que aponta para uma relação causal positiva entre os instrumentos de eficácia do governo e o desenvolvimento humano. Segundo Mauro (1995) e Gupta, Davoodi e Alonso-Terme (2002), governos eficazes podem impactar positivamente no desenvolvimento porque oferecem serviços públicos de melhor qualidade, estimulam o desenvolvimento do capital humano, incentivam a inovação tecnológica e aumentam a produtividade dos gastos do governo, possibilitando estabilidade política e sistemas burocráticos eficientes.

A qualidade regulatória é a única variável significativa que apresentou impacto individual negativo sobre o desenvolvimento humano em todas as simulações. O resultado mostrou que, para uma variação de $1 \%$ nessa variável, haverá uma variação entre-0,05\% e-0,02\% no desenvolvimento humano dos países da América Latina.

A variável Estado de Direito, que considera a proteção ao direito de propriedade e possibilita a independência dos governos e da justiça, apresentou-se significativa nas duas primeiras especificações, a um nível de $5 \%$ e 10\%. O crescimento de 1\% dessa variável acarreta acréscimos no desenvolvimento humano da ordem de 0,01\% a 0,03\%.

Adicionalmente vale ressaltar que o crescimento da renda, representada pelo PIB total e PIB per capita, mostrou efeito significativo a $1 \%$ sobre o desempenho do desenvolvimento humano dos países analisados, apesar de apresentar valores substancialmente pequenos. Esse pequeno impacto é compreensível, visto que o IDH já possui em sua composição uma proxy de renda.

Por fim, nas estatísticas apresentadas, as variáveis Voz e Responsabilização e Controle da Corrupção não se mostraram estatisticamente significantes para explicar o desempenho do desenvolvimento humano na América Latina. A análise de Campbell (2013) conclui que os indicadores de corrupção não apresentam efeitos diretos sobre o crescimento econômico e que economias imaturas podem apresentar crescimento robusto mesmo na ausência de eficiência e informação. Infere-se que o desenvolvimento humano, que possui a renda como uma de suas três dimensões, também não sofra influência direta da corrupção.

\section{CONSIDERAÇÕES FINAIS}

O presente estudo teve o objetivo de averiguar a influência das dimensões de governança no bem-estar social, por meio de indicadores globais de governança do Banco Mundial (WGI) e do índice desenvolvimento humano (IDH) dos países latino-americanos, no período de 2000 a 2014. Com base nos estudos de Wolfensohn (1998), Kaufmann, Kraay e Zoido-Lobatón (1999), Yang (2010), Gaygisiz (2013) e Sturm (2013), testou-se a hipótese de que indicadores de governança 
mundial afetam positivamente o desempenho do desenvolvimento humano dos países da América Latina. Nesse contexto, a metodologia de dados em painel, via efeitos aleatórios, atendeu ao objetivo principal e aos propósitos empíricos estabelecidos neste estudo.

O índice de desenvolvimento humano apresentou uma trajetória uniforme de crescimento, com melhor desempenho de Guatemala e Venezuela. Contudo foi observada uma tendência de menores crescimentos em países mais bem ranqueados nessa variável, como Uruguai, México, Panamá e Argentina. Não obstante, a análise do PIB e do PIB per capita também evidenciou que México e Argentina pertenceram ao grupo de países com o menor crescimento no período.

Os resultados da regressão por dados em painel foram significativos para o impacto positivo da Estabilidade Política e Ausência de Violência, da Eficácia do Governo e do Estado de Direito (exceto para a análise com o PIB total), sobre o crescimento do IDH. O crescimento das economias da região também mostrou ser um dos fatores motivadores do crescimento do Índice de Desenvolvimento humano na área de estudo. Ao mesmo tempo, observou-se também que a Qualidade Regulatória impacta negativamente no desenvolvimento humano dos países latino-americanos.

Por fim, como visto, na revisão teórica e nas estimativas aferidas, foi possível observar a importante relação entre a governança e o desenvolvimento socioeconômico na América Latina, consideradas as características específicas de cada país. Os resultados evidenciam os importantes impactos das boas práticas de governança na gestão de recursos públicos, alertando os gestores para práticas administrativas modernas aplicadas às políticas, da sua elaboração a sua fiscalização.

\section{REFERÊNCIAS}

ARELLANO, Manuel. Panel data econometrics: advanced texts in econometrics. Oxford: Oxford University Press, 2003.

BALTAGI, Badi. Econometric analysis of panel data. 3. ed. Chichester: John Wiley \& Sons, 2005.

CAMPBELL, Kaycea. A statistical analysis of public sector corruption and economic growth. LUX: A Journal of Transdisciplinary Writing and Research from Claremont Graduate University, Claremont, v. 2, n. 1, p. 6, 2013.

COMISSÃO ECONÔMICA PARA A AMÉRICA LATINA E CARIBE. Panorama social da América Latina 2014. Santiago: Cepal, 2015. Disponível em: http://repositorio.cepal.org/bitstream/handle/11362/37626/6/ S1420729_es.pdf. Acesso em: 21 dez. 2016

COMISSÃO SOBRE GOVERNANÇA GLOBAL. Nossa comunidade global - relatório da comissão sobre governança global. Rio de Janeiro: Editora FGV, 1996.

FINKELSTEIN, Lawrence S. What is global governance? Global governance, v. 1, n. 3, p. 367-72, 1995.

GAYGISIZ, Esma. How are cultural dimensions and governance quality related to socioeconomic development? The Journal of Socio-Economics, v. 47, p. 170-9, 2013.

GRINDLE, Merilee S. Good enough governance: poverty reduction and reform in developing countries. Governance, v. 17, n. 4, p. 525-48, 2004.

GUPTA, Sanjeev; DAVOODI, Hamid; ALONSO-TERME, Rosa. Does corruption affect income inequality and poverty? Economics of governance, v. 3, n. 1, p. 23-45, 2002. 
INTERNATIONAL FEDERATION OF ACCOUNTANTS (IFAC). Good Governance in the Public Sector-Consultation Draft for na International Framework. 2013. Disponível em: http://www.ifac.org/system/files/publications/ files/Good-Governance-in-the-Public-Sector.pdf. Acesso em: 30 nov. 2016.

JACQUES, Flávia V. S.; VICENTE, Ernesto F. R.; ENSSLIN, Sandra R. Indicadores de governança no setor público: bibliometria em artigos internacionais. In: CONGRESSO INTERNACIONAL DE CONTABILIDADE E AUDITORIA, 14., 10-11 out. 2013, Lisboa. Resumos [...]: Lisboa: OTOC, 2013.

JESSOP, Bob. The rise of governance and the risks of failure: the case of economic development. International social science journal, v. 50, n. 155, p. 29-45, 1998.

KAUFMANN, Daniel; KRAAY, Aart. Governance indicators: where are we, where should we be going. The World Bank research observer, v. 23, n. 1, p. 1-30, 2008.

KAUFMANN, Daniel; KRAAY, Aart; ZOIDO-LOBATÓN, Pablo. Governance Matters. World Bank policy research working paper, n. 2196. Washington, DC: World Bank, 1999.

KJAER, Anne Mette. Governance: key concepts. Cambridge, UK, 2004.

KOOIMAN, Jan; VAN VLIET, Martijn. Governance and public management. Managing public organizations, p. 58-72, 1993.

MAURO, Paolo. Corruption and growth. The quarterly journal of economics, v. 110, n. 3, p. 681-712, 1995.

MOURA, Adriana Maria Magalhães. Caminhos para uma melhor governança na política ambiental brasileira. In: Monasterio, L. M.; Neri, M. C.; Soares, S. D. S. (Org.). Brasil em desenvolvimento 2014. Brasília: IPEA, 2014. p. 103-30.

MELO NETO, Francisco Paulo; BRENNAND, Jorgiana Melo. Empresas socialmente sustentáveis: o novo desafio da gestão moderna. Rio de Janeiro: Qualitymark, 2004.

RANIS, Gustav; STEWART, Frances. Crecimiento económico y desarrollo humano en América Latina. Revista de la CEPAL, Santiago de Chile, n. 78, p. 7-24, dez. 2002.

RHODES, Rod A. W. Understanding governance: policy networks, governance, reflexivity and accountability. [S.I.]: Open university press, 1997.

SCHUMPETER, Joseph Alois. The theory of economic development: an inquiry into profits, capital, credit, interest, and the business cycle. Traduzido por Redvers Opie. Cambridge: Harvard University Press, 1961.

STOKER, Gerry. Governance as theory: five propositions. International social science journal, v. 50, n. 155, p. 17-28, 1998.

STURM, Peter. Public sector governance and development performance: an international comparison with special focus on Vietnam. DEPOCEN - Working Paper Series, n. 2013/2, 2013. Disponível em: http:// depocenwp.org/upload/pubs/2013/PeterSturm_VEAM\%20full\%20paper.pdf. Acesso em: 15 abr. 2017.

TIMMERS, Hans. Government governance: corporate governance in the public sector, why and how? In: FEE PUBLIC SECTOR CONFERENCE, 2000. Disponível em: http://www.ecgi.org/codes/documents/ public_sector.pdf. Acesso em: 14 nov. 2016.

UNITED NATIONS DEVELOPMENT PROGRAMME. Governance for sustainable human development: a UNDP policy document. UNDP, 1997. 
UNITED NATIONS DEVELOPMENT PROGRAMME. Human Development Report 2015. Nova York: Oxford University Press, 2015.

VIANA, Cristine Ferreira Gomes. Da seca como episódio à desertificação como processo: uma questão (não) institucionalizada. Orientador: Marcel Bursztyn. 2013. 244p. Tese (Doutorado em Desenvolvimento Sustentável) - Universidade de Brasília (UNB), Brasília, DF, 2013.

WEISS, Thomas G. Governance, good governance and global governance: conceptual and actual challenges. Third World Quarterly, v. 21, n. 5, p. 795-814, 2000.

WOLFENSOHN, James D. Proposal for a comprehensive development framework [for World Bank policy]: a discussion draft. Agris, 1998. Disponível em: http://agris.fao.org/agris-search/search. do?recordID=GB2013200703. Acesso em: 17 abr. 2017.

WOOLDRIDGE, Jeffrey M. Econometric analysis of cross section and panel data. 2. ed. Massachusetts: MIT press, 2010.

WORLD BANK. Can Africa claim the 21st century? Washington, DC: The World Bank, 2000.

WORLD BANK. The Worldwide Governance Indicators (WGI). World Bank, 2017a. Disponível em: http:// info.worldbank.org/governance/wgi/index.aspx\#doc. Acesso em: 30 jan. 2017.

WORLD BANK. World Development Indicators Database. World Bank, 2017b. Disponível em: http://data. worldbank.org/. Acesso em: 30 jan. 2017.

YANG, Ko-Hsin Christina. Human development and government effectiveness. Georgetown: Georgetown University, 2010.

\section{Sobre os autores:}

Francisco Aquiles de Oliveira Caetano: Mestrando em Economia Rural e graduado em Ciências Econômicas pela Universidade Federal do Ceará (UFC). Membro do Laboratório do Semiárido (LabSar). Pertencente ao Departamento de Economia Agrícola (DEA) da UFC. E-mail: aquilescaetano@yahoo.com.br

Jair Andrade de Araújo: Doutor em Economia, mestre em Economia Rural e graduado em Engenharia de Pesca pela Universidade Federal do Ceará (UFC). Coordenador do Mestrado Acadêmico em Economia Rural (MAER) do período 2014-2016. Pesquisador do grupo de pesquisa Núcleo de Estudos sobre Economia Aplicada, Pobreza e Desenvolvimento (NEAPD/MAER). Professor Adjunto da Universidade Federal do Ceará. E-mail: jairandrade@ufc.br

Ahmad Saeed Khan: Doutor em Economia Agrícola e Recursos Naturais pela Oregon State University. Mestre em Economia pela Colorado State University. Mestre em Economia Agrícola e Graduado em Agronomia pela West Pakistan Agricultural University. Professor Emérito da Universidade Federal do Ceará. E-mail: saeed@ufc.br 
